\title{
Polymer Brush-Functionalized Chitosan Hydrogels as Antifouling Implant Coatings
}

Irene Buzzacchera, ${ }^{\dagger}$ Mariia Vorobii, ${ }^{\ddagger}$ Nina Yu. Kostina, ${ }^{\ddagger}$ Andres de los Santos Pereira, ${ }^{\S}$ Tomáš Riedel, ${ }^{\S}$

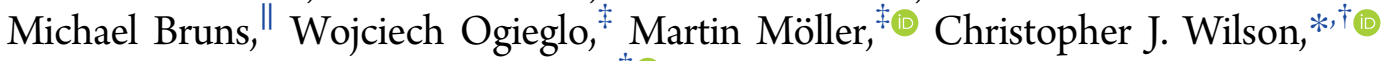
and Cesar Rodriguez-Emmenegger*, $\neq$ (1)

\author{
${ }^{\dagger}$ NovioSense B.V., Transistorweg 5, 6534 AT Nijmegen, The Netherlands \\ ${ }^{\ddagger}$ DWI-Leibniz Institute for Interactive Materials and Institute of Technical and Macromolecular Chemistry, RWTH Aachen \\ University, Forckenbeckstraße 50, 52074 Aachen, Germany \\ ${ }^{\S}$ Department of Chemistry and Physics of Surfaces and Biointerfaces, Institute of Macromolecular Chemistry, Academy of Sciences of \\ the Czech Republic, v.v.i., Heyrovsky Square 2, 16206 Prague, Czech Republic \\ "Institute for Applied Materials (IAM) and Karlsruhe Nano Micro Facility (KNMF), Karlsruhe Institute of Technology (KIT), \\ Hermann-von-Helmholtz-Platz 1, 76344 Eggenstein-Leopoldshafen, Germany
}

\section{Supporting Information}

ABSTRACT: Implantable sensor devices require coatings that efficiently interface with the tissue environment to mediate biochemical analysis. In this regard, bioinspired polymer hydrogels offer an attractive and abundant source of coating materials. However, upon implantation these materials generally elicit inflammation and the foreign body reaction as a consequence of protein fouling on their surface and concomitant poor hemocompatibility. In this report we

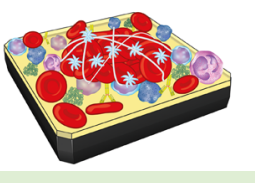
Photo SET-LRP

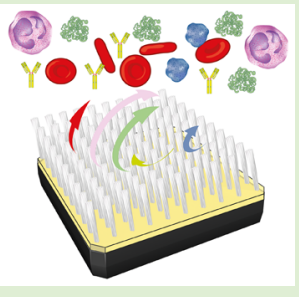
investigate a strategy to endow chitosan hydrogel coatings with antifouling properties by the grafting of polymer brushes in a "grafting-from" approach. Chitosan coatings were functionalized with polymer brushes of oligo(ethylene glycol) methyl ether methacrylate and 2-hydroxyethyl methacrylate using photoinduced single electron transfer living radical polymerization and the surfaces were thoroughly characterized by XPS, AFM, water contact angle goniometry, and in situ ellipsometry. The antifouling properties of these new bioinspired hydrogel-brush coatings were investigated by surface plasmon resonance. The influence of the modifications to the chitosan on hemocompatibility was assessed by contacting the surfaces with platelets and leukocytes. The coatings were hydrophilic and reached a thickness of up to $180 \mathrm{~nm}$ within $30 \mathrm{~min}$ of polymerization. The functionalization of the surface with polymer brushes significantly reduced the protein fouling and eliminated platelet activation and leukocyte adhesion. This methodology offers a facile route to functionalizing implantable sensor systems with antifouling coatings that improve hemocompatibility and pave the way for enhanced device integration in tissue.

\section{INTRODUCTION}

Continuous health monitoring using wearable sensors linked to cell phones is rapidly becoming a reality. For several decades, developing implantable biosensors has been a major focus of research, especially for noncommunicable diseases like diabetes. $^{1,2}$ Being able to monitor glucose subcutaneously over long periods would result in higher compliance and thus lower the cost of treatement. ${ }^{3}$ A biocompatible implantable sensor should ideally integrate and interface with surrounding tissue, facilitate the measurement of the target analyte, and maintain the required accuracy over its lifetime. ${ }^{4-6}$ Upon implantation of a foreign device inside of the human body, its surface suffers the rapid adsorption of proteins (fouling), eliciting cellular and tissue responses that isolate and attempt to degrade the foreign body ultimately diverging from natural wound healing process. The formation of a granuloma around a foreign body results in impaired fluid transport to the sensor site, leading to a significant change in sensor response over time. The first events after device implantation are protein adsorption from blood and interstitial fluids on the surface of the implant and constitution of a provisional blood matrix (thrombus). Chemical messengers such as cytokines, growth factors, and chemoattractants elicit the recruiting of innate immune system cells to the wound, triggering an acute inflammation dominated by neutrophils and lasting up to a few days. Acute inflammation turns into chronic inflammation that prevents the regular healing of tissue, as the innate immune system cells produce and release complement components, enzymes, and reactive oxygen species in their attempt to degrade and phagocytize the implant. Upon resolution of the

Received: April 10, 2017

Revised: $\quad$ May 3, 2017

Published: May 5, 2017 
inflammatory response, if macrophages fail to phagocytize implants, they fuse into multinucleated foreign body giant cells (FBGCs) triggering the foreign body reaction (FBR). FBGC secrete chemical agents responsible for degradation and induce fibroblasts to secrete collagen around the implant. Granulation tissue starts to form at the interface between the implant and tissue causing the formation of a fibrous capsule that isolates it from the body. ${ }^{6-12}$ In the case of implantable sensors, the presence of a fibrotic capsule segregating the implant from living tissue prevents fluid permeation to the sensor element resulting in an incorrect readout of the sensor. Moreover, proteins deposited on the implanted sensor surface form a "conditioning film" for bacterial attachment and proliferation, which can lead to the creation of a biofilm. Such infections are difficult to treat and put the life of the patient at risk, resulting in explant of the device. The removal of a failed implant is in many cases not a payable event and the cost is often borne by the hospital where the implantation was made.

A strategy to improve integration of implantable sensors is to embed them in extracellular matrix-like materials, in particular hydrogels that exhibit a three-dimensional network with a high content of water, mimicking physiological tissues. Materials that mimic the extracellular matrix (ECM) will potentially possess enhanced compatibility with cells due to their mechanical properties and chemical properties, which can be readily tuned to resemble those of the extracellular matrix. ${ }^{13-17}$ Various hydrogels have been used within the body, they can either be synthetic polymers such as poly(ethylene glycol) (PEG), poly(vinyl alcohol) (PVA), polyacrylamide (PAM), poly(2hydroxyethyl methacrylate) (HEMA), poly(vinylpyrrolidone) (PVP), or natural polymers such as collagen, hyaluronic acid (HA), alginate, gelatin, elastin, chondroitin sulfate, heparin, dextran, and chitosan. ${ }^{18}$ Bioinspired hydrogels based on natural materials are advantageous due to their innate biodegradability and availability from renewable sources. In particular, chitosan is a highly promising material, which can be mass-produced from nonanimal sources. ${ }^{19,20}$ Chitosan has been used in the field of drug delivery, ${ }^{21}$ as a hemostatic wound dressing, ${ }^{22-24}$ and in tissue engineering. ${ }^{25}$ The widespread use of chitosan for biomedical applications is a consequence of its welldocumented biodegradability, lack of toxicity, ${ }^{25,26}$ mucoadhesive and hemostatic properties, ${ }^{27,28}$ and intrinsic antimicrobial characteristics. $^{29}$ It consists of a cationic polysaccharide (copolymer of D-glucosamine and $\mathrm{N}$-acetyl-D-glucosamine) obtained from partial deacetylation of natural chitin. ${ }^{25,26,30}$ Chitosan hydrogels are prepared by both physical or chemical cross-linking (e.g., with glutaraldehyde). Owing to the variety of strategies available to generate chitosan hydrogels, the material properties, including swelling capacity and mechanical properties, can be tailored according to the requirements of the targeted application. ${ }^{29,31-33}$ Although chitosan in itself exhibits a low toxicity when coated onto a surface, combined with the formation of a wound on implantation, ${ }^{34}$ protein-chitosan interactions will occur leading to an inflammation response, activation of macrophages, and ultimately encapsulation of the coated device. ${ }^{35-37}$ In order to apply chitosan hydrogels as coatings on medical devices and take full advantage of their beneficial characteristics, it is necessary to modify their interface with tissues to avoid these deleterious responses. Antifouling coatings aim to suppress protein adsorption and, in this way, minimize further interactions with cellular components. ${ }^{33,38}$ In our laboratory, we have developed a strategy toward suppression of protein adsorption by utilizing densely grafted polymer brushes, achieved via a "grafting-from" methodology. The brush coatings obtained via surface-initiated living radical polymerization have shown superior resistance to nonspecific protein adsorption, even from complex biological media. Recently, their ability to prevent surface-induced thrombogenicity and adhesion of white blood cells was also established experimentally. $^{39-42}$

The aim of this work was the surface functionalization of bioinspired chitosan hydrogels with efficient antifouling synthetic polymer brushes and the characterization of the antifouling properties. Polymer brushes of poly(2-hydroxyethyl methacrylate) (poly(HEMA)) and poly[oligo(ethylene glycol) methyl ether methacrylate] (poly(MeOEGMA)) were grown from spin-coated chitosan deposited on silicon wafers functionalized with bromoisobutyrate initiator. The antifouling properties of these two synthetic polymer brushes are well-studied and reported in literature on hard surfaces. ${ }^{43}$ They have been grown by surface-initiated atom transfer radical polymerization (ATRP) on polycarbonate, ${ }^{40}$ gold, ${ }^{39,44,45}$ and glass, ${ }^{46}$ as well as polydopamine adlayers in substrate-independent approaches. ${ }^{47}$ In the present work, the polymer brushes were grown by photoinduced single electron transfer living radical polymerization (SET-LRP) directly from a "soft" chitosan surface after immobilization of a suitable initiator onto its functional groups. ${ }^{41,42,48-50}$ This novel polymerization technique allows the use of extremely low catalyst concentrations, at room temperature, and without employing aggressive solvents. $^{51,52}$ In-depth surface-physicochemical characterization showed that uniform polymer brush layers of tunable thickness could be achieved within $30 \mathrm{~min}$ of polymerization. The polymer brush-coated chitosan layers displayed excellent antifouling performance. Moreover, they effectively prevented the adhesion and activation of platelets and leukocytes. To the best of our knowledge, this is the first successful attempt in growing antifouling polymer brushes directly from spin-coated chitosan hydrogels using very mild polymerization conditions that can be directly translated into a production process for coating of a plurality of medical devices.

\section{MATERIALS AND METHODS}

Materials. Silicon wafers (orientation $\langle 100\rangle$ ) were purchased from Siegert Wafer $\mathrm{GmbH}$ (Germany). Glutaraldehyde $\left(25 \%\right.$ in $\left.\mathrm{H}_{2} \mathrm{O}\right)$, absolute ethanol ( $\geq 99.8 \%)$, triethylamine, 2-bromo 2-methylpropionyl bromide, copper(II) bromide, oligo(ethylene glycol) methyl ether methacrylate $\left(M_{\mathrm{n}}=300 \mathrm{~g} \mathrm{~mol}^{-1}\right.$, MeOEGMA) and 2-hydroxyethyl methacrylate (HEMA) were purchased from Sigma-Aldrich (Germany). The inhibitor was removed from the monomers by filtering through alumina prior to use. Chitosan $(100-300 \mathrm{kDa}$, degree of deacetylation $\geq 90 \%$ ), extra dry tetrahydrofuran (THF), and extra dry dimethyl sulfoxide (DMSO) were purchased from Acros Organics. Tris(2-dimethylaminoethyl)amine ( $\left.\mathrm{Me}_{6} \mathrm{TREN}, 99 \%\right)$ was purchased from Alfa Aesar (Germany). Acetic acid (100\%) was purchased from VWR (Germany). Human fibrinogen (Fbg), and human serum albumin (HSA) were purchased from Sigma-Aldrich (Czech Republic). Pooled human blood plasma (HBP, mix of 5 donors) was obtained from the Institute of Hematology and Blood Transfusion (Czech Republic).

Spin Coating of a Chitosan Hydrogel on Si Wafers. Si wafers were cut by diamond cutter in $1 \times 1 \mathrm{~cm}^{2}$ wafers, they were separately sonicated in ethanol in an ultrasonic bath for $10 \mathrm{~min}$ and washed twice with ethanol and Milli- $Q$ water. Wafers were dried under a stream of $\mathrm{N}_{2}$ and treated with plasma for 20 min to remove any organic impurity and generate hydroxyl groups at the surface. The coating solution was prepared mixing $3.5 \mu \mathrm{L}$ of $25 \% \mathrm{v} / \mathrm{v}$ solution of glutaraldehyde to $1 \mathrm{~mL}$ of $0.35 \% \mathrm{w} / \mathrm{v}$ solution of chitosan in $1 \%$ acetic acid. The solution was 
mixed by vortex for $30 \mathrm{~s}$ and $50 \mu \mathrm{L}$ were directly dispensed onto the plasma-treated $\mathrm{Si}$ wafer on the spin coater. The wafers were spun at $5000 \mathrm{rpm}$ for $60 \mathrm{~s}$ with an acceleration time of $5 \mathrm{~s}$. The chitosan coated wafers were stored in Milli-Q water at room temperature for 24 $\mathrm{h}$ to remove excess chitosan not bound to the surface. After $24 \mathrm{~h}$ they were carefully washed with fresh Milli-Q water and dried under a stream of $\mathrm{N}_{2}$ before the successive steps.

Initiator Attachment on Chitosan Films. The chitosan-coated wafers were immersed in $15 \mathrm{~mL}$ of a $0.24 \mathrm{M}$ solution of triethylamine in THF. A $0.24 \mathrm{M}$ solution of the initiator 2-bromo-2-methylpropionyl bromide in THF $(7.5 \mathrm{~mL})$ was added dropwise while the wafers were gently agitated. After $3 \mathrm{~min}$ of gentle agitation, the wafers were washed sequentially with THF, ethanol, Milli-Q water, and dried under a stream of $\mathrm{N}_{2}$.

Surface-Initiated Photo SET-LRP of Poly(MeOEGMA) and Poly(HEMA). A stock solution was prepared by dissolving $\mathrm{CuBr}_{2}(3.9$ $\mathrm{mM}$ ) and the ligand $\mathrm{Me}_{6}$ TREN $(23.4 \mathrm{mM})$ in DMSO. The stock solution was stirred at room temperature until complete dissolution, avoiding light exposure. The polymerization solution was prepared by dissolving $7.92 \mathrm{mmol}$ of the monomer and $200 \mu \mathrm{L}$ of stock solution in $4.5 \mathrm{~mL}$ of DMSO in a round-bottom flask wrapped in $\mathrm{Al}$ foil to prevent light exposure. The polymerization solution was degassed by bubbling with $\mathrm{N}_{2}$ for $30 \mathrm{~min}$. The wafers with the initiator immobilized on the chitosan layer were degassed separately with $\mathrm{N}_{2}$ for $15 \mathrm{~min}$ before the addition of the polymerization solution. The polymerization reaction was performed in a UV reactor equipped with a $\lambda$ 320-400 nm lamp. The polymerization was stopped at selected time points by opening the vials, diluting the polymerization solution with fresh DMSO and washing the wafers sequentially with ethanol and Milli- $\mathrm{Q}$ water. The wafers were dried under $\mathrm{N}_{2}$.

Ellipsometry. The dry thickness of the layers was measured using an OMT Ellipsometer and VisuEl software version 3.4.1 (Optische Messtechnik $\mathrm{GmbH}$ ) at the angle of incidence of $70^{\circ}$ and a spectral method with wavelength range from 460 to $870 \mathrm{~nm}$. The swelling study was performed with an RC2 spectroscopic ellipsometer from J. A. Woollam Co., Inc. using a quartz in situ cell. The modeling of the measurement was done with CompleteEASE software version 5.07. The analysis was conducted in a wavelength range 193-1000 nm using spectra of a high resolution ( 800 wavelengths per spectrum). For the swelling experiments the cell window offsets as well as the influence of the ambient liquid on the signal were taken into account.

X-ray Photoelectron Spectroscopy (XPS). Surface-chemical characterization was performed with a K-Alpha+ XPS spectrometer (Thermo Fisher Scientific, East Grinstead, U.K.). The samples were analyzed using a microfocused, monochromated $\mathrm{Al} \mathrm{K} \alpha \mathrm{X}$-ray source ( $400 \mu \mathrm{m}$ spot size). The kinetic energy of the electrons was measured using a $180^{\circ}$ hemispherical energy analyzer operated in the constant analyzer energy mode (CAE) at $50 \mathrm{eV}$ pass energy for elemental spectra. The spectra were fitted with one or more Voigt profiles (binding energy uncertainty: $\pm 0.2 \mathrm{eV}$ ). The analyzer transmission function, Scofield sensitivity factors, ${ }^{53}$ and effective attenuation lengths (EALs) for photoelectrons were applied for quantification. EALs were calculated using the standard TPP-2 M formalism. ${ }^{54}$ All spectra were referenced to the $\mathrm{C} 1 \mathrm{~s}$ peak of hydrocarbons at $285.0 \mathrm{eV}$ binding energy controlled by means of the well-known photoelectron peaks of metallic $\mathrm{Cu}, \mathrm{Ag}$, and $\mathrm{Au}$.

Atomic-Force Microscopy. All AFM images were acquired with a Multimode Atomic Force Microscope NanoScope V (Digital Instruments) as topological scans in tapping mode in air, using silicon probes PPP-SEIH-10 (NANOSENSORS) with a nominal spring constant of $15 \mathrm{~N} \cdot \mathrm{m}^{-1}$ and a tip radius of $10 \mathrm{~nm}$. Areas of $5 \times 5 \mu \mathrm{m}^{2}$ $(512 \times 512$ pixels $)$ were scanned at a rate of $1 \mathrm{~Hz}$. The phase images were obtained by monitoring the phase lag of the cantilever vibration compared to the z-piezo drive voltage while the probe scans the surface with a preset constant amplitude of vibration. The phase data contain additional information about the tip-sample interactions resulting from adhesion, surface stiffness, and viscoelastic effects. The scans were analyzed using Gwyddion software.

Assessment of the Antifouling Properties by Surface Plasmon Resonance (SPR). Nonspecific protein adsorption (fouling) was measured on chitosan-coated surfaces before and after growth of the polymer layer via SPR spectroscopy using an instrument based on the Kretschmann geometry and spectral interrogation (Institute of Photonics and Electronics, Czech Republic). Solutions were driven through a flow cell by a peristaltic pump at a flow rate of $25 \mu \mathrm{L} \cdot \mathrm{min}^{-1}$ and the SPR responses were measured as shifts in the resonant wavelength, $\lambda_{\text {res }}$. First, a baseline was established in phosphate buffered saline (PBS, pH 7.4). Solutions in PBS of Fbg $\left(1 \mathrm{mg} \mathrm{mL}^{-1}\right)$ or HSA ( $\left.5 \mathrm{mg} \mathrm{mL}^{-1}\right)$, and HBP (10\% in PBS) were subsequently flowed over the surface for $15 \mathrm{~min}$, after which the flowing solution was replaced with PBS. The sensor response $\left(\Delta \lambda_{\text {res }}\right)$ was obtained as the difference between the baselines in PBS before and after the injection of the analyzed solutions and was converted to surface mass coverage using a calibration made previously $\left(\Delta \lambda_{\text {res }}=1 \mathrm{~nm}\right.$ corresponds to an increase in the deposited protein mass of $15 \mathrm{ng}$ $\mathrm{cm}^{-2}$ ).

Dynamic Water Contact Angle. The wettability of the surfaces was assessed by measuring the dynamic water contact angle using the sessile drop method with a DSA100 instrument from Krüss GmbH (Germany). A $5 \mu \mathrm{L}$ drop was placed on the surface and its volume was increased up to $15 \mu \mathrm{L}$ and decreased back to $5 \mu \mathrm{L}$ at a flow rate of 0.2 $\mu \mathrm{L} \mathrm{s}^{-1}$. The drop profile was fitted with a circular algorithm, from which the advancing and receding contact angles were obtained.

Hemocompatibility Test. The surfaces used for this study consisted of $\mathrm{Si}$ wafer coated with chitosan films by spin coating, either bare or functionalized with polymer brushes. To test the hemocompatibility of the poly(MeOEGMA) and poly(HEMA) brushes grafted from chitosan, the surfaces were incubated with platelet-rich plasma (PRP) and leukocytes isolated from fresh blood. Bare chitosan surfaces were used as a control. All individuals tested agreed to this study at the time of blood collection. All samples were obtained in accordance with the Ethical Committee regulations of the Institute of Hematology and Blood Transfusion, Prague, and with release of informed consent. Platelets and leukocytes were obtained according to literature. ${ }^{39}$

A $200 \mu \mathrm{L}$ drop of PRP or leukocytes was deposited on the surface to be tested and incubated for $60 \mathrm{~min}$ at $37^{\circ} \mathrm{C}$. Subsequently, the surfaces were thoroughly washed with $0.9 \%$ saline; fixed by glutaraldehyde $(0.5 \%)$ for $60 \mathrm{~min}$, rinsed with water and then with ethanol, and allowed to dry. The dried surfaces were sputter coated with $4 \mathrm{~nm}$ platinum and observed using scanning electron microscopy (SEM). Images were evaluated using Image data analysis software. The number of adherent platelets and leukocytes was determined by calculating the surface coverage of individual components. Three independent spots were analyzed for each sample. The studies were carried out in triplicate.

\section{RESULTS AND DISCUSSION}

Chitosan Films Preparation and Stability. The polymer brushes were grown from ultrathin chitosan hydrogel films. The hydrogels were prepared on plasma-cleaned silicon wafers by spin coating of a mixture of chitosan (dissolved in $1 \%$ acetic acid solution, $\mathrm{pH} 2.8$ ) to which glutaraldehyde (cross-linker) is added immediately prior spinning. After spin coating, the hydrogels were incubated for $24 \mathrm{~h}$ in Milli-Q water to remove any excess chitosan not covalently bound. The film thickness was measured by ellipsometry in the dry state at three different points on the surface, with a measured area of $2 \mathrm{~mm}^{2}$ for each point. The films were homogeneous, with an average thickness of $12 \pm 0.5 \mathrm{~nm}$. Chitosan is a high molecular weight natural polymer only soluble at low $\mathrm{pH}$ in aqueous conditions. As the molecular weight of the chains increases due to glutaraldehyde cross-linking, it becomes insoluble. The stable deposition of the noncovalently bound film on the $\mathrm{Si}$ surface results from increased insolubility of the high molecular weight cross-linked chains and the noncovalent interactions occurring at the interface between the hydrogel and the $\mathrm{Si}$ wafer. To assess the 
stability of the layers, the coated wafers were stored in Milli-Q water at room temperature for $52 \mathrm{~d}$ and the evolution of the film thickness was followed in dry state, after careful washing of the samples with Milli-Q water (Figure 1). Generally, the

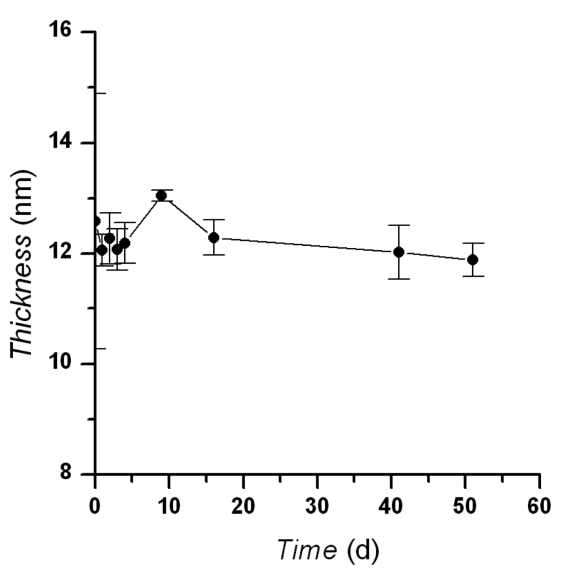

Figure 1. Average thickness of five chitosan films on Si wafers stored in Milli-Q water at room temperature for $52 \mathrm{~d}$. The thickness was measured by ellipsometry on rinsed and dried samples. The error bars indicate the standard deviation for every point.

thickness varied within a range of $1 \mathrm{~nm}$, which confirms that the deposited films are very stable in the $52 \mathrm{~d}$ period. The stability of the films is an essential condition for the grafting of polymer brushes.

The expected chemical structure of the chitosan film on the surfaces was verified by X-ray photoelectron spectroscopy (XPS; Figure 2a,c). The high-resolution spectrum of the $\mathrm{C} 1 \mathrm{~s}$ region shows the predominance of the component at $286.4 \mathrm{eV}$, which arises from $\mathrm{C}-\mathrm{O}$ abundant in the carbohydrate structure. The contribution of $\mathrm{C}-\mathrm{N}$ forming the amine groups is overlapped and not resolved. The component at $288.0 \mathrm{eV}$ originates from amide groups, indicating that the deacetylation is not complete. The $\mathrm{N} 1 \mathrm{~s}$ spectrum of the chitosan film reveals the predominance of amine groups (neutral at $399.3 \mathrm{eV}$ and protonated at $402.4 \mathrm{eV}$ ) with respect to the amide groups (at $400.1 \mathrm{eV}$ ). These observations confirm the abundance of hydroxyl and amine groups that can be addressed to immobilize the initiator in an acylation reaction.

Photoinduced SET-LRP of Polymer Brushes and Surface Characterization. Essential to a graft-from-surface living radical polymerization is the presence of an alkyl halide initiator covalently attached to the surface. A common initiator group for SET-LRP consisting of 2-bromo-2-methylpropionate was used. ${ }^{55,56}$ The chitosan-coated Si wafers were treated with a solution of the initiator in THF in basic conditions, an acylation reaction with the functional groups of chitosan ensures covalent linkage of the initiator to the hydrogel (Scheme 1). XPS measurements corroborated the immobilization of the initiator species (Figure $2 \mathrm{~b}$ ). The initiator moieties gave rise to a spinorbit coupled doublet in the $\mathrm{Br} 3 \mathrm{~d}$ region of the spectrum of the initiator-modified chitosan layer.

The chitosan surfaces treated with 2-bromo-2-methylpropionyl bromide were used as the initiating site for grafting of poly(MeOEGMA) or poly(HEMA) by photoinduced SETLRP. Polymer brush films with thickness of up to 180 and 50 $\mathrm{nm}$ were obtained, respectively, in less than $30 \mathrm{~min}$. The chemical structure of the surfaces and the presence of the different polymer brushes on the chitosan layers were
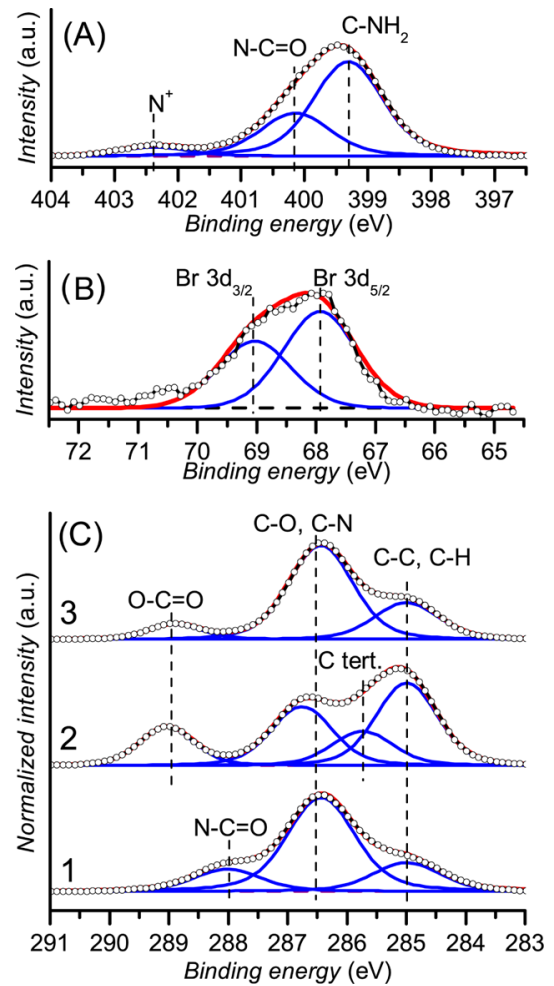

Figure 2. High-resolution XPS spectra of (A) N 1s region of the asdeposited chitosan layer; (B) $\mathrm{Br} 3 \mathrm{~d}$ region of the initiatorfunctionalized chitosan layer; and $(\mathrm{C}) \mathrm{C}$ 1s region of the as-deposited chitosan layer (1), poly(HEMA) (2), and poly(MeOEGMA) (3). All peak assignments are in a good agreement with literature values. ${ }^{39,57}$

confirmed by XPS (Figure 2c). The high-resolution spectra of the $\mathrm{C} 1 \mathrm{~s}$ region clearly show the features expected from the grafted polymer brushes. Notably, both the brushes of poly(HEMA) and poly(MeOEGMA) display a contribution at $289.0 \mathrm{eV}$, which arises from the ester moiety in the methacrylate backbone. Carbon atoms forming $\mathrm{C}-\mathrm{O}$ bonds give rise to a component appearing at 286.8 and $286.4 \mathrm{eV}$ for poly(HEMA) and poly(MeOEGMA), respectively. In the latter case, this contribution predominates in the spectrum due to the abundance of $\mathrm{C}-\mathrm{O}$ units in the oligo(ethylene glycol) side chain. In the case of poly(HEMA), the contribution of the tertiary carbon in the methacrylate backbone appears resolved at $285.7 \mathrm{eV}$, as a result of secondary shift from the ester group. No signals are visible in the $\mathrm{N}$ 1s spectra of poly(HEMA) and poly(MeOEGMA) as expected (Figure S1). It is worth noting that the XPS spectra of the surfaces modified with polymer brushes lack the spectral features of the chitosan layer. This confirms that the polymer layers are thick enough to cover the chitosan film and attenuate its signals (over 8-10 nm). ${ }^{54}$

In general, the kinetic study of surface-initiated polymerizations presents a number of challenges not least of which is the inaccessibility of utilizing typical solution phase methods to analyze the resulting polymer. Furthermore, the variation in the monomer concentration in solution is a poor indicator of kinetic profile of the reaction as the monomer conversion is negligible due to the extremely low amount of initiating species in the chitosan film compared to the monomer concentration. With this in mind, the indicative rate of the polymerization was investigated by measuring the increase in thickness of the polymeric layer grown onto chitosan over time, as it is directly proportional to the rate of propagation. ${ }^{58,59}$ However, it should 


\section{Scheme $1^{a}$}

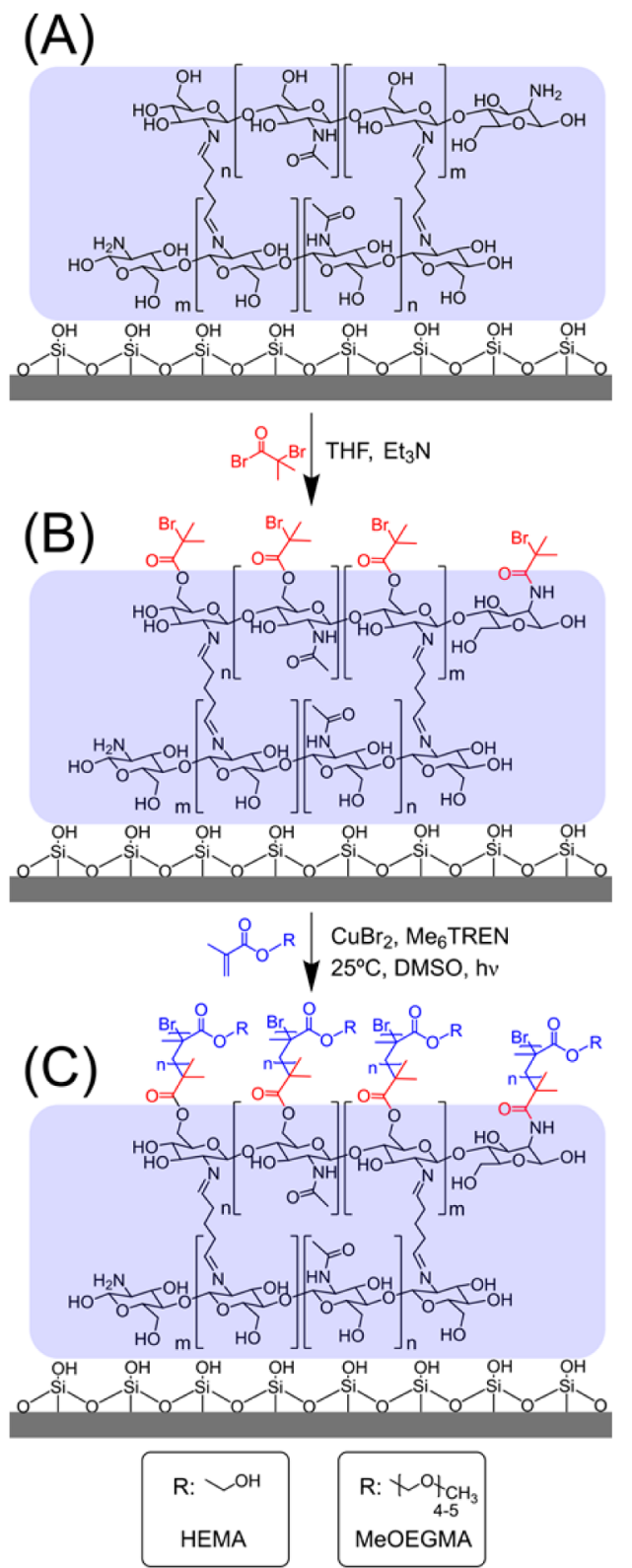

${ }^{a}$ Illustration of the chitosan hydrogel deposited on top of Si wafers and stabilized by weak interactions between the chitosan monomers and the $\mathrm{OH}$ groups on the surface of the $\mathrm{Si}$ wafers (A). The initiator reacts by acylation with the free functional groups of the chitosan monomers (B). The polymer brushes are grown from the chitosan hydrogel functionalized with the initiator (C). HEMA: 2-hydroxyethyl methacrylate; MeOEGMA: oligo(ethylene glycol) methyl ether methacrylate $\left(M_{\mathrm{n}}=300 \mathrm{~g} \mathrm{~mol}^{-1}\right)$.

be noted that the thickness does not yield any information about the molecular weight distribution, other than showing an increase in the average molecular weight $M_{\mathrm{w}}$. The thickness of the polymer brushes was measured by ellipsometry in dry state at three different points of the surface. The thickness values obtained for each samples differed on average less than 3-4 $\mathrm{nm}$. By plotting the dry polymer thickness vs polymerization time, a linear tendency is obtained, indicating that the average rate of polymerization over the surface is constant during the reaction. This is consistent with the growing polymerization centers being preserved to a large extent and suggests that termination processes are probably minor (Figure 3). Nevertheless, assessment of the whole molecular weight distribution

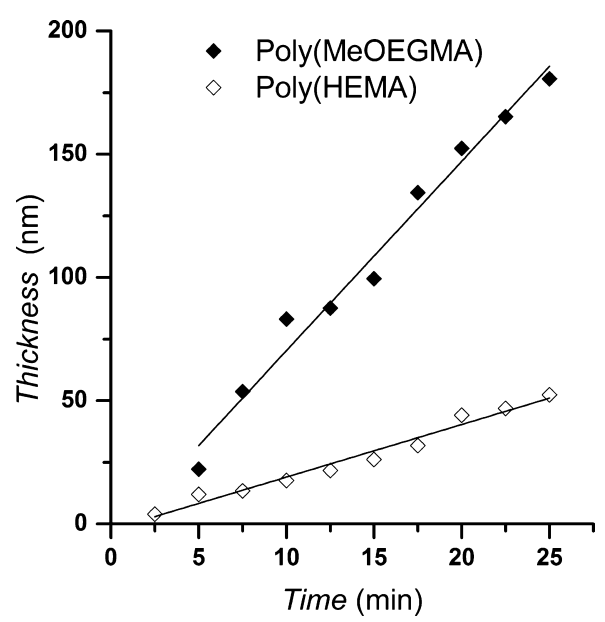

Figure 3. Dry thickness of the polymer brushes grown on chitosan thin films versus polymerization time. The rate of thickness growth of poly(MeOEGMA) $(\diamond)$ is $7.7 \pm 0.41 \mathrm{~nm} \times \min ^{-1}$ and for poly(HEMA) $(\diamond)$ is $2.1 \pm 0.12 \mathrm{~nm} \times \mathrm{min}^{-1}$. The polymerization was performed at $25{ }^{\circ} \mathrm{C}$ under UV light exposure $(\lambda 320-400 \mathrm{~nm})$, monomer concentration $1.76 \mathrm{M}, \mathrm{CuBr}_{2} 3.9 \mathrm{mM}, \mathrm{Me}_{6}$ TREN $23.4 \mathrm{mM}$.

and end group fidelity would be required to completely rule out the possibility of early termination processes. The polymers show different indicative rates of polymerization, the slope from the linear fitting is $7.7 \pm 0.41 \mathrm{~nm} \times \min ^{-1}$ for poly(MeOEGMA) and $2.1 \pm 0.12 \mathrm{~nm} \times \mathrm{min}^{-1}$ for poly(HEMA). Assuming the reactivities of the methacrylate polymerizable groups are similar in both monomers, the observed difference in the rates of thickness growth can be partly explained by the bigger size of the MeOEGMA monomer in comparison to HEMA (having a $2.3 \times$ higher molecular weight), so that the propagation step adds more mass to the polymer layer in the case of MeOEGMA.

The topography of the surfaces was studied by AFM. Comparisons of the images obtained for bare chitosan, the polymer brushes on chitosan, and $\mathrm{Si}$ controls are shown in Figures 4 and S2. Both the Si wafer substrate and the bare chitosan film were smooth, as seen in the corresponding images and indicated by very low root-mean-square roughness values, $R_{\mathrm{q}}$, of 0.64 and $0.25 \mathrm{~nm}$ for bare chitosan and $\mathrm{SiO}_{2}$, respectively. Following polymerization, the height images for both polymer layers grafted from chitosan show homogeneous coverages, while the $R_{\mathrm{q}}$ roughness values were slightly higher than for bare chitosan at 1.2 and $0.7 \mathrm{~nm}$ for poly(HEMA) and poly(MeOEGMA), respectively. No pinhole defects are visible, which is a necessary requirement for effective prevention of protein adsorption. Surprisingly, poly(HEMA) showed an increase in the phase images, probably indicating a more viscoelastic surface, which was more likely to be expected in poly(MeOEGMA). The more viscoelastic behavior of the poly(HEMA)-functionalized layers might be associated with differences in the architecture of the films. It is conceivable, the smaller HEMA monomer could allow the growth from inside the chitosan layer, while the larger MeOEGMA only from the interface. This could lead to different mechanical and optical properties (refer to ellipsometry section).

Moreover, the overall uniformity observed within the AFM phase images confirms the homogeneity of the surface 


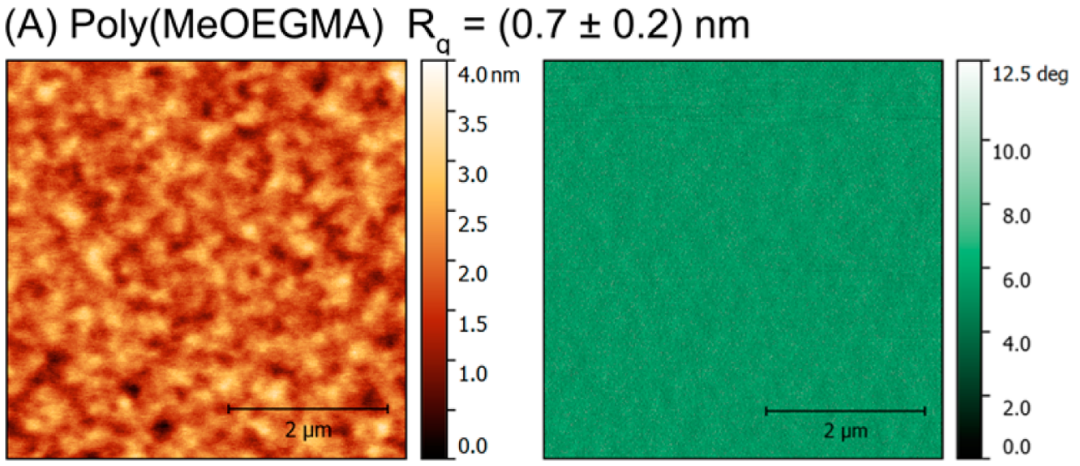

(B) Poly(HEMA) $R_{q}=(1.2 \pm 0.2) n m$
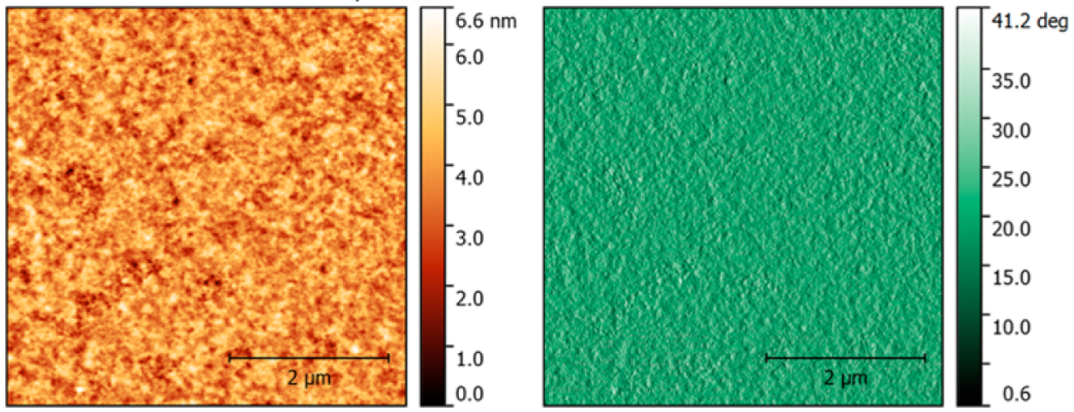

Figure 4. AFM height (left) and phase (right) topography images of chitosan functionalized with poly(MeOEGMA) (A) and chitosan functionalized with poly(HEMA) (B) on Si wafers. The scale bar is $2 \mu \mathrm{m}$, the root mean-square roughness $R_{\mathrm{q}}$ is reported: (A) $R_{\mathrm{q}}=(0.7 \pm 0.2) \mathrm{nm}$, total thickness of the film $40 \mathrm{~nm}$; (B) $R_{\mathrm{q}}=(1.2 \pm 0.2) \mathrm{nm}$, total thickness of the film $50 \mathrm{~nm}$.

coverage. Thus, the polymerization procedure used enables the growth of dense and uniform polymeric layers on top of the chitosan hydrogel base coating.

The wettability of the surfaces was investigated by measuring the advancing and receding water contact angles. Functionalization of the chitosan hydrogel films with polymer brushes did not alter the hydrophilic character of the layers, seen especially in the low values of the receding contact angles. Interestingly, all surfaces show rather large contact angle hysteresis values $\left(\Delta \theta=\theta_{\mathrm{a}}-\theta_{\mathrm{b}} ;\right.$ Table 1$)$. This phenomenon is probably caused

Table 1. Dynamic Water Contact Angles of the Surfaces

\begin{tabular}{lcc} 
& \multicolumn{2}{c}{ water contact angle $(\mathrm{deg})$} \\
\cline { 2 - 3 } \multicolumn{1}{c}{ surface } & advancing, $\theta_{\mathrm{a}}$ & receding, $\theta_{\mathrm{b}}$ \\
chitosan & $60 \pm 3$ & $9 \pm 2$ \\
poly(MeOEGMA) on chitosan & $57 \pm 1$ & $9 \pm 2$ \\
poly(HEMA) on chitosan & $61 \pm 1$ & $14 \pm 1$ \\
\hline
\end{tabular}

by the swelling of the chitosan and polymer layers, as well as reorientation of the hydrophilic functional groups during wetting of the surface.

Characterization of the Swelling Behavior Using In Situ Ellipsometry. The swelling behavior of chitosan and chitosan functionalized with polymer brushes was measured in PBS buffer at room temperature with in situ spectroscopic ellipsometry. For this we determined $\Psi$ and $\Delta$ in the dry state as well as after equilibration with the buffer. The study of the swelling of ultrathin films combined with precise optical modeling of the film gave us access for the first time to the hierarchical structure of the swollen multilayer films. The optical properties of pure components, necessary for the subsequent precise modeling of the swollen multilayer films were determined on spin-coated chitosan and poly(HEMA) and poly(MeOEGMA) directly grown from silicon wafers modified with 11-(2-bromoisobutyryl)undecyltrichlorosilane. $\Psi$ and $\Delta$ were recorded at broad spectral $(192-1000 \mathrm{~nm})$ and angular (AOI: $55-80^{\circ}$ every $1^{\circ}$ ) ranges followed by B-Splinebased fit of both real and imaginary parts (complex refractive index: $N=n+i \cdot k)$ of the dielectric function. ${ }^{60}$ In the B-Spline fit, first a Cauchy layer is used to fit the approximate layer thickness and the real part of the complex refractive index in the visible range (where the sample can be considered transparent). Then the layer is converted to B-Spline and the model is extended to include the ultraviolet part of the spectrum, thus determining the imaginary part of the complex refractive index (associated with light absorption). The resulting optical properties of the pure components are shown in Supporting Information (Figure S3). The obtained refractive indices at $\lambda=632.8 \mathrm{~nm}$ were 1.501 for poly(MeOEGMA), 1.515 for poly(HEMA), and 1.533 for chitosan. The rather similar refractive indices of the three materials result in poor optical contrast in the visible range making it difficult to model a stack of chitosan and brushes. To enhance the contrast and to differentiate between brushes and chitosan we utilized the UV absorption of chitosan at $\lambda \sim 300 \mathrm{~nm}$, which is not present in the polymer brushes. For the determination of the thickness in the dry state of the brushes on chitosan, the films were approximated with a two-layer optical model utilizing the $\mathrm{UV}$ absorption of chitosan to discriminate it from the brushes. The total thicknesses were $41.6 \mathrm{~nm}$ for poly(HEMA) on chitosan and $48.7 \mathrm{~nm}$ for poly(MeOEGMA) on chitosan. The thicknesses of each individual layer were determined to be 18.0 $\mathrm{nm}$ poly(HEMA) on $23.6 \mathrm{~nm}$ chitosan and $35.5 \mathrm{~nm}$ poly(MeOEGMA) on $13.2 \mathrm{~nm}$ chitosan. While the total thicknesses can be considered of very high accuracy, the values of the thicknesses of individual layers heavily rely on the assumption of a sharp optical interface between the two layers. 
The modeled chitosan thickness in the poly(MEOEGMA) sample is close to its value before brush growth $(16.6 \mathrm{~nm})$, suggesting that poly(MeOEGMA) brushes predominantly grew from the interface. On the other hand, the thickness of the chitosan layer underneath poly(HEMA) is considerably higher than the thickness of the same chitosan layer measured before the grafting of the brushes $(16.3 \mathrm{~nm})$, suggesting that the poly(HEMA) simultaneously grew from interface and inside the chitosan layer, thus, modifying and smearing out the position of the optical interface. The smaller monomer size of HEMA probably allows for the growth deeper within the chitosan layer. When the brush-functionalized samples were equilibrated in PBS, a large difference in the overall swelling factor $\left(\mathrm{SF}=\frac{h_{\text {swollen }}}{h_{\text {dry }}}\right)$ of both samples could be seen. For the poly(MeOEGMA) grown from chitosan the total swollen thickness was much larger, approximately $171 \mathrm{~nm}$ giving an overall $\mathrm{SF}=3.5$. For this sample a two-layer model was found to be numerically valid and unique. The detailed analysis, including fit parameter correlations and uniqueness fits, is shown in Supporting Information (Figure S4 and Table S2). The swollen thicknesses of the chitosan layer and the poly(MeOEGMA) layers were found to be 71 and $100 \mathrm{~nm}$, respectively. Using the previously ellipsometry-derived dry chitosan thickness $(13.2 \mathrm{~nm})$, the SF of the chitosan layer alone equals to 5.4, which is much larger than for a nonfunctionalized chitosan, $\mathrm{SF}=1.55$. This pronounced increase in the SF could be ascribed to the forces exerted by the brushes onto the chitosan film caused by the osmotic pressure and entropic effects arising from the stretched polymer chain conformation. The SF for poly(MeOEGMA) layer on chitosan is found to be 2.8, which is larger than that of the same brushes directly grown from silicon $(\mathrm{SF}=1.9)$, suggesting that the grafting density of brushes on chitosan might be lower than that of brushes directly grown from a sharp rigid interface. Since poly(HEMA) grew from inside and from the interface of chitosan a two-layer model was deemed not valid and instead a graded optical model was utilized. A graded optical model assumes a continuous variation of the sample refractive index orthogonal to the substrate. The choice of the graded model was substantiated by a large reduction of the fit error parameter (mean square error, MSE) going from a uniform single layer model to graded single layer model $\left(\mathrm{MSE}_{\text {uniform }}=17\right.$ to $\mathrm{MSE}_{\text {graded }}=9$ ). The resulting total swollen thickness was $74 \mathrm{~nm}$ and $\mathrm{SF}=1.8$. The latter is considerably lower than for the more hydrophilic poly(MeOEGMA)-functionalized chitosan. After recalculation using the effective medium approximation ${ }^{61}$ and a typical refractive index for the system, it was found that the bottom of the sample possessed a slightly higher water volume fraction than the top (approximately 60\% vs $40 \%$ ). This may suggest a larger degree of swelling within the underlying chitosan structure than within the poly(HEMA) brush residing on the top.

Assessment of Antifouling Properties of Polymer Brushes on Chitosan. Protein adsorption was investigated by surface plasmon resonance (SPR) on the prepared surfaces. Chitosan films were prepared on gold chips for SPR by spincoating and they were functionalized with polymer brushes using the same synthetic procedures as on Si. The thickness of the chitosan layers was about $10 \mathrm{~nm}$ and for the polymer brush coatings a thickness of approximately $20 \mathrm{~nm}$ was selected based on previous results of fouling resistance on model surfaces. ${ }^{62,63}$ Four model solutions of fibrinogen (Fbg, $1 \mathrm{mg} \mathrm{mL}^{-1}$ ), human

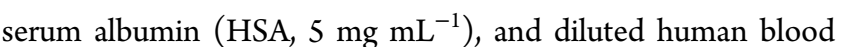
plasma (HBP, $10 \%$ in PBS) were put in contact with the prepared films. Importantly, bare chitosan suffers rapid adsorption of $\mathrm{Fbg}$ when contacted with a single-protein solution at a concentration similar to human plasma (Figure 6). This protein is important in the coagulation cascade,

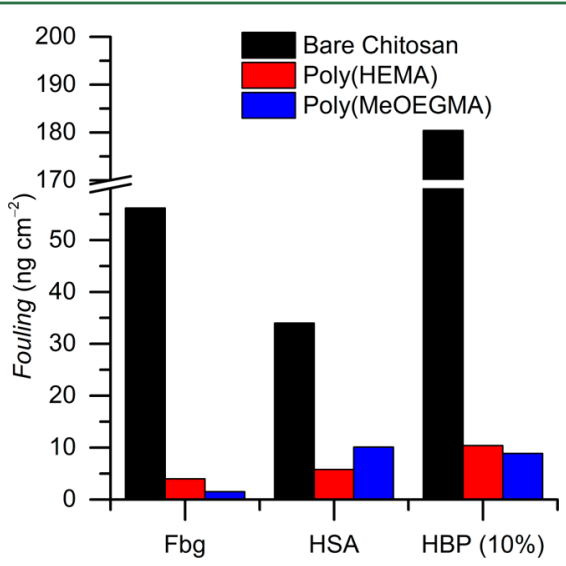

Figure 6. Fouling on chitosan, chitosan functionalized with poly(MeOEGMA), and chitosan functionalized with poly(HEMA) in

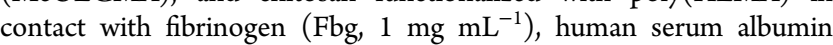
(HSA, $5 \mathrm{mg} \mathrm{mL}^{-1}$ ), and diluted human blood plasma (HBP, 10\% in PBS). The fouling was determined using SPR after 15 min contact.

mediating the aggregation of activated platelets, and is negatively charged at physiological $\mathrm{pH}$, thus, it is attracted by the surface due to the weak cationic character of chitosan. Upon adsorption, intact Fbg molecules can undergo conformational changes revealing cryptic integrin binding sites for platelets and leukocytes. ${ }^{64}$ Thus, this protein also plays an early central role in the triggering of surface thrombogenicity and inflammation. Importantly, both poly(HEMA) and poly(MeOEGMA) were able to reduce the fouling from Fbg by $93 \%$ and $97 \%$, respectively. This represents a significant improvement in comparison with previously presented chitosan coating methods, owing to the dense brush architecture achieved via the grafting-from approach. ${ }^{65}$ Moreover, the coating of chitosan with poly(HEMA) and poly(MeOEGMA) reduced the adsorption of HSA, the most abundant protein in plasma, by $83 \%$ and $70 \%$, respectively. Nevertheless, resistance to the adsorption of individual proteins from simple solutions in buffer does not imply the ability to prevent fouling from complex biological fluids, which represent a much more demanding test. ${ }^{66,67}$ For this purpose, fouling from HBP at $10 \%$ in PBS was assessed. The antifouling properties of the polymer brush-coated chitosan layers were evidenced by the marked reduction in fouling from this challenging medium, showing a decrease of $94 \%$ for poly(HEMA) and $95 \%$ for poly(MeOEGMA) in comparison with bare chitosan, which experienced rapid protein fouling in this medium (please refer to Figure S5 for the SPR sensograms showing the adsorption kinetics).

Hemocompatibility of Chitosan before and after Modification. Assessment of the suitability of bare chitosan and polymer brush-coated chitosan films for tissue-contact applications was performed in vitro by characterizing the attachment and activation of important blood cellular components on their surface, namely, leukocytes and platelets (from platelet-rich plasma, PRP). Cells normally present in 
blood were selected because this fluid is typically the first medium that a device implanted in tissue will come into contact within the course of surgery. Importantly, platelets are the major player in the thrombogenic response, as well as secreting chemical messengers playing a role in the inflammation pathway. On the other hand, leukocytes mediate the inflammatory response, thus, being central to the wound healing process. Adhesion of erythrocytes was not analyzed, as previous work showed it to be negligible, and this type of cell has not been implicated in body responses threatening implant hemocompatibility. ${ }^{39}$

Figure 7 shows typical SEM micrographs obtained from the surfaces after $60 \mathrm{~min}$ of contact with the cells at $37^{\circ} \mathrm{C}$, rinsing

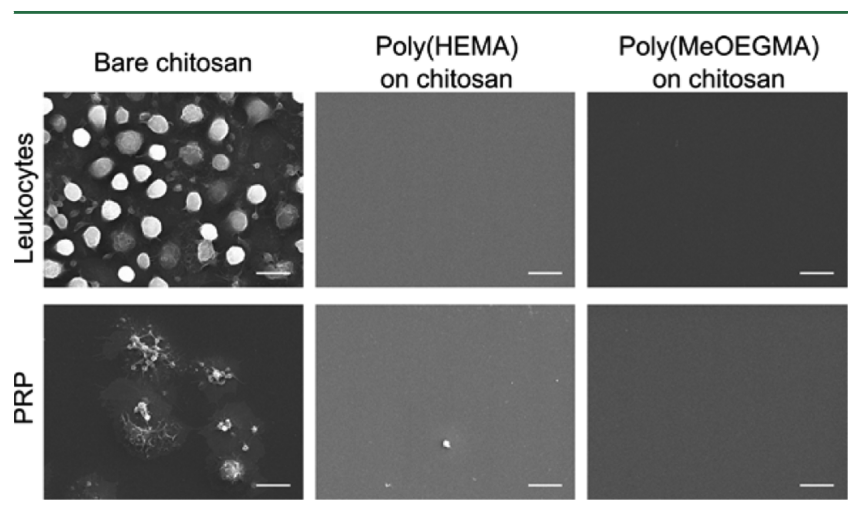

Figure 7. Attachment of leukocytes (top row) and platelets (from platelet-rich plasma, PRP, bottom row) on bare chitosan (left column), poly(HEMA)-coated chitosan (middle column), and poly(MeOEGMA)-coated chitosan (right column), as analyzed via SEM after contact. In all images, the scale bar represents $10 \mu \mathrm{m}$.

with buffer, fixation with glutaraldehyde, and drying from ethanol. The chitosan surface shows massive adhesion of large leukocytes, showing various degrees of spreading. The smaller cells adhering to the surface are platelets remaining in the suspension after isolation of the leukocytes. Upon close examination of the background, an extremely dense surface coverage of completely spread leukocytes is observed. Quantitative analysis of the images indicates that the area covered by the cells amounts to $94.0 \%$ (Figure 8). This

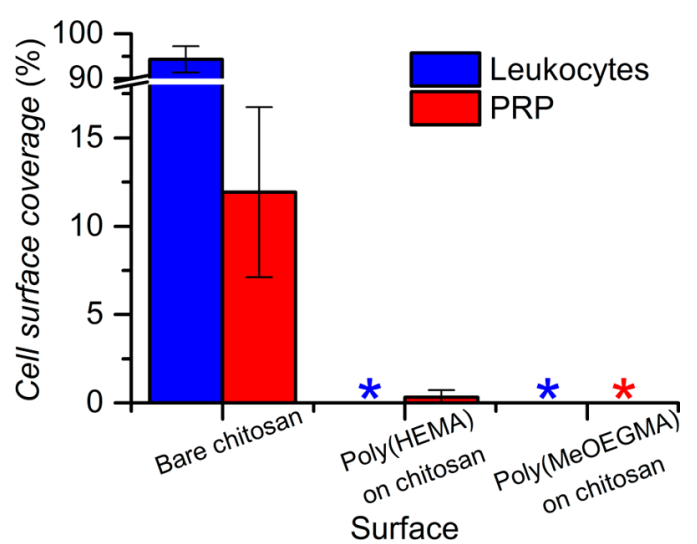

Figure 8. Comparison of the surface coverage on the bare chitosan and poly(HEMA)- or poly(MeOEGMA)-coated chitosan after $60 \mathrm{~min}$ contact with a leukocyte suspension or PRP at $37{ }^{\circ} \mathrm{C}$. Note: the symbol "*” means that no cells were detected on the surface, thus, the surface coverage was 0 . suggests that implantation of bare chitosan hydrogels would result in the rapid triggering of the inflammatory response and eventually favor the FBR, leading to formation of a fibrotic capsule around the implant, as well as impaired wound healing. In sharp contrast, when the chitosan surface was coated with poly(HEMA) or poly(MeOEGMA) brushes, adhesion of leukocytes was fully prevented. This highlights the potential of these layers to prevent inflammatory responses, thus enhancing the integration of chitosan-coated devices in tissue. Upon contact of bare chitosan with PRP, extensive adhesion of platelets is observed on its surface, amounting to a surface coverage of $11.9 \%$. This value is similar to values previously reported for bare gold. ${ }^{39}$ Importantly, these platelets show clear signs of activation, as their morphology is highly distorted from the unperturbed discoidal shape displaying significant spreading on the substrate.

Platelet activation triggers the release of coagulation factors from their granulocytes and creates additional highly prothromobgenic area on the surface of the cells themselves, setting the coagulation cascade in motion. As a consequence, the activated platelets recruit additional platelets from the medium, resulting in aggregation, as seen in the image, leading to concomitant amplification of the coagulation response. It is important to note that coating of the chitosan surface with poly(MeOEGMA) fully prevents the adhesion of platelets, thus, eliminating surface thrombogenicity. Poly(HEMA)-coated chitosan shows the presence of a small amount of platelets on the surface (a reduction of $97 \%$ with respect to bare chitosan). Nevertheless, these cells appear individually (i.e., nonaggregated) and retain their lenticular shape, indicating that they are not activated. Thus, they are not expected to contribute greatly to surface thrombogenicity, in agreement with our previous reports on the hemocompatibility of polymer brushes under flow conditions. ${ }^{40}$ The ability of polymer brush coatings on chitosan to repel the attachment of blood cells is therefore consistent with previous results, which were focused on a model substrate and indicated greatly improved hemocompatibility. ${ }^{39}$ In this regard, polymer brushes are able not only to prevent the fouling by plasma proteins (which leads to blood cell adhesion and activation), but also the direct surface attachment of the blood cells themselves. The rejection of these interactions is critical, as they lead to adverse biological responses hindering wound healing and implant-tissue integration.

\section{CONCLUSIONS}

In this report, we have set out to develop a system to grow antifouling polymer brushes from a bioinspired chitosan hydrogel as a coating for implantable biosensing devices with improved integration into tissue. The method utilizes readily accessible monomers and base coating materials that are employed in existing medical products and a functionalization strategy is based on photoinduced SET-LRP, requiring a low catalyst loading and mild conditions. Chitosan hydrogel coatings showed high surface uniformity and the brushes grown from the polysaccharide substrate were hydrophilic and both uniform and pinhole-free. In situ ellipsometry showed that the grafting of a poly(MeOEGMA) brush increased the swelling in buffer of the underlying chitosan hydrogel. On the other hand, the grafting of poly(HEMA) proceeded partially inside the chitosan hydrogel and simultaneously as a brush grafted from the interface. In contrast to bare chitosan, the polymer brush coatings showed excellent resistance to protein 
fouling. Importantly, antifouling polymer brushes were able to prevent the adhesion and activation of blood cellular components on the chitosan coatings, thus, showing enhanced hemocompatibility. This report demonstrates a facile route to antifouling coating systems that can be rapidly industrialized using available monomers and base coatings and deployed into existing medical products as well as in a new generation of implantable sensor systems.

\section{ASSOCIATED CONTENT}

\section{S Supporting Information}

The Supporting Information is available free of charge on the ACS Publications website at DOI: 10.1021/acs.biomac.7b00516.

Stability of chitosan films on Si wafers stored in Milli-Q water at room temperature for $52 \mathrm{~d}$, additional XPS spectra of the polymer layers on chitosan, AFM topography and phase images of silicon and chitosan on silicon wafer, optical properties of the pure components used for the modeling, and fit evaluation and parameter correlations of the ellipsometry model (PDF).

\section{AUTHOR INFORMATION}

\section{Corresponding Authors}

*E-mail: rodriguez@dwi.rwth-aachen.de.

*E-mail: c.wilson@noviosense.com.

\section{ORCID}

Andres de los Santos Pereira: 0000-0002-0138-1357

Martin Möller: 0000-0002-5955-4185

Christopher J. Wilson: 0000-0003-0760-3421

Cesar Rodriguez-Emmenegger: 0000-0003-0745-0840

Notes

The authors declare no competing financial interest.

\section{ACKNOWLEDGMENTS}

C.W., M.M., and I.B. gratefully acknowledge that this project has received funding from the European Union's Horizon 2020 research and innovation programme under the Marie Sklodowska-Curie grant agreement No 642687. T.R. and A.d.l.S.P. are grateful for the support from the Grant Agency of the Czech Republic (GACR) under Contract No. P205-12G118. This work was partly carried out with the support of the Karlsruhe Nano Micro Facility (KNMF).

\section{REFERENCES}

(1) Carrara, S.; Ghoreishizadeh, S.; Olivo, J.; Taurino, I.; Baj-Rossi, C.; Cavallini, A.; de Beeck, M. O.; Dehollain, C.; Burleson, W.; Moussy, F. G.; Guiseppi-Elie, A.; De Micheli, G. Sensors 2012, 12 (12), 11013-11060.

(2) Kotanen, C. N.; Moussy, F. G.; Carrara, S.; Guiseppi-Elie, A. Biosens. Bioelectron. 2012, 35 (1), 14-26.

(3) Klonoff, D. C. J. Diabetes Sci. Technol. 2007, 1 (6), 797-800.

(4) Ratner, B. D. J. Cardiovasc. Transl. Res. 2011, 4 (5), 523-527.

(5) Ratner, B. D.; Hoffman, A. S.; Schoen, F. J.; Lemons, J. E. Biomaterials Science: An Introduction to Materials in Medicine, 2nd ed.; Elsevier Academic Press: New York, NY, 2004.

(6) Koh, A.; Nichols, S. P.; Schoenfisch, M. H. J. Diabetes Sci. Technol. 2011, 5 (5), 1052-1059.

(7) Sheikh, Z.; Brooks, P.; Barzilay, O.; Fine, N.; Glogauer, M. Materials 2015, 8 (9), 5671-5701.

(8) Major, M. R.; Wong, V. W.; Nelson, E. R.; Longaker, M. T.; Gurtner, G. C. Plast. Reconstr. Surg. 2015, 135 (5), 1489-1498.
(9) Junge, K.; Binnebösel, M.; von Trotha, K. T.; Rosch, R.; Klinge, U.; Neumann, U. P.; Lynen Jansen, P. Langenbeck Arc. Surg. 2012, 397 (2), 255-270.

(10) Bryers, J. D.; Giachelli, C. M.; Ratner, B. D. Biotechnol. Bioeng. 2012, 109 (8), 1898-1911.

(11) Franz, S.; Rammelt, S.; Scharnweber, D.; Simon, J. C. Biomaterials 2011, 32 (28), 6692-6709.

(12) Anderson, J. M.; Rodriguez, A.; Chang, D. T. Semin. Immunol. 2008, 20 (2), 86-100.

(13) Wilson, C. J.; Akeroyd, Z. H. E.; Rowan, A. E.; Feitz, W. F. J.; Geutjes, P. J.; Oosterwijk, E. Polymer suitable for use in cell culture. EP3021872, 2016.

(14) Zhu, J.; Marchant, R. E. Expert Rev. Med. Devices 2011, 8 (5), 607-626.

(15) Caló, E.; Khutoryanskiy, V. V. Eur. Polym. J. 2015, 65, 252-267.

(16) Ng, V. W. L.; Chan, J. M. W.; Sardon, H.; Ono, R. J.; García, J. M.; Yang, Y. Y.; Hedrick, J. L. Adv. Drug Delivery Rev. 2014, 78, 46-62.

(17) Salomé Veiga, A.; Schneider, J. P. Biopolymers 2013, 100 (6), 637-644.

(18) Annabi, N.; Tamayol, A.; Uquillas, J. A.; Akbari, M.; Bertassoni, L. E.; Cha, C.; Camci-Unal, G.; Dokmeci, M. R.; Peppas, N. A.; Khademhosseini, A. Adv. Mater. 2014, 26 (1), 85-123.

(19) Dodane, V.; Vilivalam, V. D. Pharm. Sci. Technol. Today 1998, 1 (6), 1-8.

(20) Ilium, L. Pharm. Res. 1998, 15 (9), 1326-1331.

(21) Luo, Y.; Wang, Q. Int. J. Biol. Macromol. 2014, 64, 353-367.

(22) Pogorielov, M. V.; Sikora, V. Z. Europ. J. Med., B 2015, 2 (1), 24-33.

(23) Ghobril, C.; Grinstaff, M. W. Chem. Soc. Rev. 2015, 44 (7), 1820-1835.

(24) Rieger, K. A.; Birch, N. P.; Schiffman, J. D. J. Mater. Chem. B 2013, 1 (36), 4531-4541.

(25) Dash, M.; Chiellini, F.; Ottenbrite, R. M.; Chiellini, E. Prog. Polym. Sci. 2011, 36 (8), 981-1014.

(26) Bellich, B.; D’Agostino, I.; Semeraro, S.; Gamini, A.; Cesàro, A. Mar. Drugs 2016, 14 (5), 99-31.

(27) Mathews, S.; Kaladhar, K.; Sharma, C. P. J. Biomed. Mater. Res., Part A 2006, 79A (1), 147-152.

(28) Sagnella, S.; Mai-Ngam, K. Colloids Surf., B 2005, 42 (2), 147155 .

(29) Hosseinnejad, M.; Jafari, S. M. Int. J. Biol. Macromol. 2016, 85, 467-475.

(30) Pillai, C. K. S.; Paul, W.; Sharma, C. P. Prog. Polym. Sci. 2009, 34 (7), 641-678.

(31) Ahmadi, F.; Oveisi, Z.; Samani, S. M.; Amoozgar, Z. Res. Pharm. Sci. 2015, 10 (1), 1-16.

(32) Shukla, S. K.; Mishra, A. K.; Arotiba, O. A.; Mamba, B. B. Int. J. Biol. Macromol. 2013, 59, 46-58.

(33) Harding, J. L.; Reynolds, M. M. Trends Biotechnol. 2014, 32 (3), 140-146.

(34) Berscht, P. C.; Nies, B.; Liebendörfer, A.; Kreuter, J. J. Mater. Sci.: Mater. Med. 1995, 6 (4), 201-205.

(35) Peluso, G.; Petillo, O.; Ranieri, M.; Santin, M.; Ambrosic, L.; Calabró, D.; Avallone, B.; Balsamo, G. Biomaterials 1994, 15 (15), $1215-1220$

(36) Chen, C.-L.; Wang, Y.-M.; Liu, C.-F.; Wang, J.-Y. Biomaterials 2008, 29 (14), 2173-2182.

(37) Nishimura, K.; Ishihara, C.; Ukei, S.; Tokura, S.; Azuma, I. Vaccine 1986, 4 (3), 151-156.

(38) Chen, S.; Li, L.; Zhao, C.; Zheng, J. Polymer 2010, 51 (23), $5283-5293$.

(39) Surman, F.; Riedel, T.; Bruns, M.; Kostina, N. Y.; Sedláková, Z.; Rodriguez-Emmenegger, C. Macromol. Biosci. 2015, 15 (5), 636-646.

(40) de los Santos Pereira, A.; Sheikh, S.; Blaszykowski, C.; PopGeorgievski, O.; Fedorov, K.; Thompson, M.; RodriguezEmmenegger, C. Biomacromolecules 2016, 17 (3), 1179-1185.

(41) Laun, J.; Vorobii, M.; de los Santos Pereira, A.; Pop-Georgievski, O.; Trouillet, V.; Welle, A.; Barner-Kowollik, C.; Rodriguez- 
Emmenegger, C.; Junkers, T. Macromol. Rapid Commun. 2015, 36 (18), 1681-1686.

(42) Vorobii, M.; de los Santos Pereira, A.; Pop-Georgievski, O.; Kostina, N. Y.; Rodriguez-Emmenegger, C.; Percec, V. Polym. Chem. 2015, 6 (23), 4210-4220.

(43) Banerjee, I.; Pangule, R. C.; Kane, R. S. Adv. Mater. 2011, 23 (6), 690-718.

(44) Vaisocherová, H.; Ševců, V.; Adam, P.; Špačková, B.; Hegnerová, K.; de los Santos Pereira, A.; Rodriguez-Emmenegger, C.; Riedel, T.; Houska, M.; Brynda, E.; Homola, J. Biosens. Bioelectron. 2014, 51, $150-157$.

(45) Zhao, C.; Li, L.; Wang, Q.; Yu, Q.; Zheng, J. Langmuir 2011, 27 (8), 4906-4913.

(46) Mrabet, B.; Nguyen, M. N.; Majbri, A.; Mahouche, S.; Turmine, M.; Bakhrouf, A.; Chehimi, M. M. Surf. Sci. 2009, 603 (16), 24222429.

(47) Pop-Georgievski, O.; Rodriguez-Emmenegger, C.; de los Santos Pereira, A.; Proks, V.; Brynda, E.; Rypáček, F. J. Mater. Chem. B 2013, 1 (22), 2859-2867.

(48) Zhang, N.; Samanta, S. R.; Rosen, B. M.; Percec, V. Chem. Rev. 2014, 114 (11), 5848-5958.

(49) Rosen, B. M.; Percec, V. Chem. Rev. 2009, 109 (11), 50695119.

(50) Lligadas, G.; Grama, S.; Percec, V. Biomacromolecules 2017, 18 (4), 1039-1063.

(51) Percec, V.; Guliashvili, T.; Ladislaw, J. S.; Wistrand, A.; Stjerndahl, A.; Sienkowska, M. J.; Monteiro, M. J.; Sahoo, S. J. Am. Chem. Soc. 2006, 128, 14156-14165.

(52) Percec, V.; Popov, A. V.; Ramirez-Castillo, E.; Monteiro, M.; Barboiu, B.; Weichold, O.; Asandei, A. D.; Mitchell, C. M. J. Am. Chem. Soc. 2002, 124, 4940-4941.

(53) Scofield, J. H. J. Electron Spectrosc. Relat. Phenom. 1976, 8 (2), 129-137.

(54) Tanuma, S.; Powell, C. J.; Penn, D. R. Surf. Interface Anal. 2005, 37 (1), 1-14.

(55) Anastasaki, A.; Nikolaou, V.; Zhang, Q.; Burns, J.; Samanta, S. R.; Waldron, C.; Haddleton, A. J.; McHale, R.; Fox, D.; Percec, V.; Wilson, P.; Haddleton, D. M. J. Am. Chem. Soc. 2014, 136 (3), 11411149.

(56) Samanta, S. R.; Nikolaou, V.; Keller, S.; Monteiro, M. J.; Wilson, D.; Haddleton, D. M.; Percec, V. Polym. Chem. 2015, 6, 2084-2097.

(57) Rodriguez-Emmenegger, C.; Janel, S.; de los Santos Pereira, A.; Bruns, M.; Lafont, F. Polym. Chem. 2015, 6 (31), 5740-5751.

(58) Matyjaszewski, K.; Miller, P. J.; Shukla, N.; Immaraporn, B.; Gelman, A.; Luokala, B. B.; Siclovan, T. M.; Kickelbick, G.; Vallant, T.; Hoffmann H, A.; Pakula, T. Macromolecules 1999, 32 (26), 87168724.

(59) Tomlinson, M. R.; Kirill Efimenko, A.; Genzer, J. Macromolecules 2006, 39 (26), 9049-9056.

(60) Johs, B.; Hale, J. S. Phys. Status Solidi A 2008, 205 (4), 715-719.

(61) Bruggeman, D. A. G. Ann. Phys. 1935, 416 (7), 636-664.

(62) Rodriguez-Emmenegger, C.; Avramenko, O. A.; Brynda, E.; Skvor, J.; Alles, A. B. Biosens. Bioelectron. 2011, 26 (11), 4545-4551.

(63) Vorobii, M.; Pop-Georgievski, O.; de los Santos Pereira, A.; Kostina, N. Y.; Jezorek, R.; Sedláková, Z.; Percec, V.; RodriguezEmmenegger, C. Polym. Chem. 2016, 7 (45), 6934-6945.

(64) Lishko, V. K.; Kudryk, B.; Yakubenko, V. P.; Yee, V. C.; Ugarova, T. P. Biochemistry 2002, 41, 12942-12951.

(65) Wang, X.; Yuan, S.; Guo, Y.; Shi, D.; Jiang, T.; Yan, S.; Ma, J.; Shi, H.; Luan, S.; Yin, J. Colloids Surf., B 2015, 136, 7-13.

(66) Emmenegger, C. R.; Brynda, E.; Riedel, T.; Sedlakova, Z.; Houska, M.; Alles, A. B. Langmuir 2009, 25 (11), 6328-6333.

(67) Blaszykowski, C.; Sheikh, S.; Thompson, M. Chem. Soc. Rev. 2012, 41 (17), 5599-5612. 